\title{
Left mediastinal node dissection after arterial ligament transection via video-assisted thoracoscopic surgery for potentially advanced stage I non-small cell lung cancer
}

\author{
Tomoki Shibano, Hiroyoshi Tsubochi, Kenji Tetsuka, Shinichi Yamamoto, Yoshihiko Kanai, \\ Kentaro Minegishi, Shunsuke Endo
}

Department of General Thoracic Surgery, Jichi Medical University, Shimotsuke-shi, Tochigi, Japan

Contributions: (I) Conception and design: S Endo; (II) Administrative support: H Tsubochi; (III) Provision of study materials or patients: S Endo; (IV) Collection and assembly of data: T Shibano; (V) Data analysis and interpretation: T Shibano; (VI) Manuscript writing: All authors; (VII) Final approval of manuscript: All authors.

Correspondence to: Shunsuke Endo, MD, PhD. Department of General Thoracic Surgery, Jichi Medical University, Yakushiji, Shimotsuke-shi, Tochigi 329-0498, Japan. Email: tcvshun@jichi.ac.jp.

\begin{abstract}
Background: Left mediastinal node dissection during lung cancer surgery can be difficult because paratracheal and subcarinal lymph nodes are concealed by mediastinal structures. Arterial ligament transection (ALT) offers a wide surgical view of concealed mediastinal spaces, thus enabling extended en bloc lymph node dissection (LND). We analyzed surgical outcomes of patients who underwent extended LND after ALT via video-assisted thoracoscopic surgery (VATS) for potentially node-positive clinical stage I nonsmall cell lung cancer (NSCLC).

Methods: We retrospectively investigated the medical records of 75 patients who had undergone extended mediastinal node dissection after ALT via VATS for potentially node-positive NSCLC at our centers during the period from September 2008 through November 2015. Operative data and rates of overall survival (OS), in addition to mortality and morbidity, were analyzed in relation pathological stage and nodal stage.

Results: Operative time was $238 \pm 58$ minutes, and an average of $32.7 \pm 12.9$ hilar and mediastinal lymph nodes were dissected. Lymph node metastases were detected in 34 patients $(6 \mathrm{pN} 1$ patients, $27 \mathrm{pN} 2$ patients, and $1 \mathrm{pN} 3$ patient). Mediastinal lymph node metastases were detected around the carina (stations $2 \mathrm{~L}, 4 \mathrm{~L}$, and 7) in 19 of 27 patients with $\mathrm{pN} 2$ cancer. Nineteen patients had a total of 24 postoperative complications. Recurrent nerve paralysis was the most frequent complication $(\mathrm{n}=11)$ but resolved in eight patients during follow-up. Survival rates at 3 and 5 years were $92.2 \% / 88.4 \%, 100.0 \% / 60.0 \%$, and $87.7 \% / 81.0 \%$ for p-stage I, II, and III, respectively, and $92.2 \% / 88.4 \%, 100.0 \% / 60.0 \%$, and $87.4 \% / 80.7 \%$ for $\mathrm{pN} 0$, pN1, and $\mathrm{pN} 2$, respectively.
\end{abstract}

Conclusions: Extended mediastinal node dissection after ALT allowed detection of lymph node micrometastases in selected patients with potentially node-positive left NSCLC and may improve outcomes.

Keywords: Lung cancer; nodal dissection; arterial ligament; micrometastasis; video-assisted thoracoscopic surgery (VATS)

Submitted Jul 31, 2018. Accepted for publication Nov 05, 2018.

doi: $10.21037 /$ jtd.2018.11.86

View this article at: http://dx.doi.org/10.21037/jtd.2018.11.86

\section{Introduction}

Systematic mediastinal lymph node dissection (LND) or sampling of non-small cell lung cancer (NSCLC) is strongly recommended in published guidelines $(1,2)$. However, mediastinal LND around the carina is anatomically challenging because mediastinal structures conceal the 
left paratracheal lymph nodes (stations $2 \mathrm{~L}$ and $4 \mathrm{~L}$ ) and subcarinal lymph nodes (station 7).

Here, we describe the thoracoscopic procedures and surgical results of extended mediastinal LND involving transection of the arterial ligament for treatment of potentially node-positive left NSCLC. This technique offers a wide thoracoscopic view of the bilateral paratracheal and subcarinal spaces and facilitates detection of lymph node micrometastases in selected patients with cancer of the left lung and potential node involvement.

\section{Methods}

\section{Study design and patients}

This retrospective study was conducted at Jichi Medical University, Tochigi, Japan, and Jichi Saitama Medical Center, Saitama, Japan, from September 2008 through November 2015. Data were collected from the medical records of 75 patients who had undergone arterial ligament transection (ALT) during mediastinal systematic nodal dissection via video-assisted thoracoscopic surgery (VATS) for potentially node-positive clinical stage I NSCLC. This study was approved by the institutional review board of Jichi Medical University (No. 17-056). Informed consent was obtained in the form of opt-out on a website for the study.

\section{Surgical indications}

The patients with clinical stage I NSCLC, as categorized by the criteria included in the 7th Edition of the International Association for the Study of Lung Cancer (3) were enrolled in this study. Clinical stage was determined on the basis of preoperative chest computer tomography (CT) findings. All patients underwent positron emission tomography (PET)-CT before surgery. Potentially node-positive stage I NSCLC was diagnosed when at least one of the following factors was present:

(I) The standardized uptake value (SUV) of ${ }^{18} \mathrm{~F}$-fluorodeoxyglucose in the primary tumor was greater than 3 on preoperative PET ( $n=63$ ).

(II) The SUV of ipsilateral nodes was higher than that of contralateral nodes $(n=12)$. Invasive biopsy via bronchoscopy was not performed because the longest diameter of lymph nodes was less than $7 \mathrm{~mm}$.

(III) Preoperative serum carcinoembryonic antigen (CEA) concentration was greater than the normal cut-off value of $5 \mathrm{ng} / \mathrm{dL}(\mathrm{n}=22)$.
(IV) Hilar or mediastinal metastasis was suggested by intraoperative pathological analysis $(n=28)$.

\section{Surgery}

Patients were placed in a right lateral decubitus position. All surgeries were successfully performed by 5 -portaccess thoracoscopic surgery with a rigid $45^{\circ}$ scope. After anatomical lung resection (segmentectomy or lobectomy) and systematic LND of the hilar, mediastinal lymph node stations $3 \mathrm{a}, 5$, and 6 (zone 1), the arterial ligament was exposed and dissected with an ultrasonic energy device. The main operator performed en bloc dissection of the lymph nodes around the carina (zone 2: stations $2 \mathrm{~L}, 4 \mathrm{~L}$, and 7 ) while an assistant maintained a wide aorticopulmonary window (Figure 1).

\section{Pathological diagnosis}

The numbers of dissected and metastatic lymph nodes were counted by the surgeons and pathologists. Pathological diagnoses were made by at least two pathologists. TNM staging was determined by using criteria specified in the 7 th Edition of the International Association for the Study of Lung Cancer.

\section{Statistical analysis}

Statistical analyses were performed with the SPSS statistical software package (version 25.0, IBM, Armonk, NY, USA). Differences between groups were evaluated with the twosample $t$-test, for continuous variables, and the Chisquare test, for categorical variables. Overall survival (OS) was evaluated by Kaplan-Meier analysis, and differences between groups were analyzed with the log-rank test. A P value of 0.05 or less was considered to indicate statistical significance.

\section{Results}

\section{Surgical results}

Thirty-seven men and 38 women were included in the analysis (mean age $63.4 \pm 7.3$ years) (Table 1). Left upper lobectomy was the most frequent surgical procedure performed ( $\mathrm{n}=57)$, followed by left lower-lobe lobectomy $(\mathrm{n}=15)$ and segmentectomy $(\mathrm{n}=3)$. Mean operative time was $238 \pm 58$ minutes and intraoperative blood loss was 


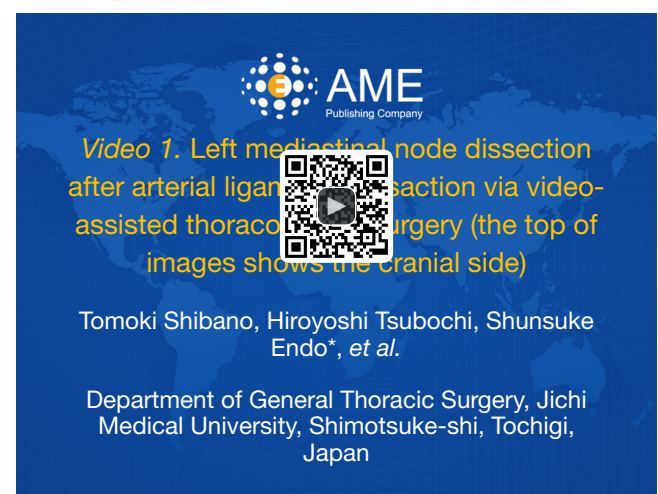

Figure 1 Left mediastinal node dissection after arterial ligament transaction via video-assisted thoracoscopic surgery (the top of images shows the cranial side) (4). The patient was a 71-year-old man with left NSCLC. Clinical staging was T1bN0M0, serum CEA concentration was $6.7 \mathrm{ng} / \mathrm{mL}$, and PET-SUV was 14. The patient was placed in the right decubitus position under general anesthesia. Three ports were placed at the 5 th intercostal space, one at the anterior axillary line at the 3rd intercostal space, and one at the posterior axillary line at the 7 th intercostal space. The procedures were successfully performed under 5 -port-access thoracoscopic surgery using a rigid $45^{\circ}$ scope. The interlobar pulmonary artery is exposed. The mediastinal pleura is opened along the phrenic nerve with scissors and marked with rubber tape. The hilar lymph nodes are dissected, and the superior vein, left upper bronchus, and anterior segmental artery (in that order) are divided with an automatic suturing device. After completion of the left upper lobectomy, the anterior mediastinal lymph nodes (station 3a, 5, 6) are dissected along with left thymic tissues. The aortic arch, left brachiocephalic vein, and left phrenic and vagal nerves are identified during the dissection. The arterial ligament is taped and transected with a harmonic scalpel (Ethicon Endo-Surgery, Cincinnati, OH, USA). Lymph nodes are dissected from the left main bronchus, cranially, to the bifurcation of the pulmonary artery by ventrally towing the left main pulmonary artery. When necessary, scissors and hemostatic clips are used around the recurrent nerve, to avoid paralysis. The mediastinal site of paratracheal lymph nodes is dissected until the left edge of superior vena cava, and the frontal section of the carina is completely exposed. The right trachea-bronchial lymph nodes are then dissected. The cranial side of subcarinal lymph nodes is separated from the carina by caudally towing the left main pulmonary artery. The left atrium, bilateral main bronchus, and esophagus are located at the peripheral edge of the subcarinal lymph nodes. Subcarinal dissection is completed by cranially towing the left main pulmonary artery. Operative time was 215 minutes, and blood loss was $165 \mathrm{~mL}$. Pathological analysis confirmed adenocarcinoma with invasion of the visceral pleura. Sixty lymph nodes were dissected, but no metastases were identified. Postoperative course was uneventful, and the patient was discharged on day 10 after surgery. NSCLC, non-small cell lung cancer; CEA, carcinoembryonic antigen; PET, positron emission tomography; SUV, standardized uptake value.

Available online: http://www.asvide.com/article/view/29193

$179 \pm 162 \mathrm{~mL}$ (Table 2). There were no intraoperative complications related to ALT. Twenty-four postoperative complications occurred in 19 patients. The most frequent was hoarseness due to recurrent nerve paralysis $(n=11)$, followed by arrhythmia $(n=7)$, pneumonia $(n=2)$, emboli of the superior mesenteric artery $(\mathrm{n}=1)$, empyema $(\mathrm{n}=1)$, chylothorax $(n=1)$, and prolonged air leak $(n=1)$. There were no in-hospital deaths (Table 3). Eight of the 11 patients $(72.7 \%)$ with hoarseness recovered during followup (Table 2). Routine bronchoscopy was performed for every patient on 7 th postoperative day, because ischemic bronchitis was at high risk for extended LND. Thus, median duration of hospitalization was 12 days. Duration of hospitalization was 33, 75, and 132 days for patients with empyema, pneumonia, and emboli of the superior mesenteric artery postoperatively, respectively.

\section{Pathological results}

The pathological diagnoses were adenocarcinoma $(\mathrm{n}=65)$, squamous cell carcinoma $(\mathrm{n}=8)$, large-cell carcinoma $(\mathrm{n}=1)$, and typical carcinoid tumor $(n=1)$. An average of $32.7 \pm 12.9$ hilar and mediastinal lymph nodes were dissected. Lymph node metastasis was detected in 34 patients (6 classified as $\mathrm{pN} 1 ; 27$ as $\mathrm{pN} 2$ ). Metastasis to the right paratracheal lymph nodes was noted in 1 patient (with $\mathrm{pN} 3$ cancer). The average number of metastatic lymph nodes was $5.00 \pm 5.05$ in node-positive patients $(\mathrm{pN} 1,1.33 \pm 0.51 ; \mathrm{pN} 2$, 5.81 $\pm 5.34 ; \mathrm{pN} 3,5)$. Among the 27 patients with $\mathrm{pN} 2$ cancer, mediastinal lymph node metastasis was present at 
Table 1 Patient characteristics

\begin{tabular}{|c|c|c|}
\hline Variable & Total $n$ & Value/percentage \\
\hline Age (years), mean \pm SD & 75 & $63.4 \pm 7.3$ \\
\hline \multicolumn{3}{|l|}{ Sex } \\
\hline Male & 37 & $49 \%$ \\
\hline Female & 38 & $51 \%$ \\
\hline \multicolumn{3}{|l|}{ Smoking history } \\
\hline Never smoker & 33 & $44 \%$ \\
\hline Past or current smoker & 42 & $56 \%$ \\
\hline Pack-years, median [range] & & $38[5-180]$ \\
\hline \multicolumn{3}{|l|}{ Histologic type } \\
\hline Adenocarcinoma & 65 & $87 \%$ \\
\hline Squamous cell carcinoma & 8 & $11 \%$ \\
\hline Large cell carcinoma & 1 & $1 \%$ \\
\hline Carcinoid & 1 & $1 \%$ \\
\hline \multicolumn{3}{|l|}{ Cancer localization } \\
\hline Upper lobe & 59 & $79 \%$ \\
\hline Lower lobe & 16 & $21 \%$ \\
\hline \multicolumn{3}{|l|}{ Pathological nodal status } \\
\hline pNO & 41 & $55 \%$ \\
\hline $\mathrm{pN} 1$ & 6 & $8 \%$ \\
\hline pN2 & 27 & $36 \%$ \\
\hline pN3 & 1 & $1 \%$ \\
\hline Tumor size $(\mathrm{mm})$, mean \pm SD & 75 & $26.6 \pm 8.6$ \\
\hline SUVmax, mean \pm SD & 75 & $6.5 \pm 3.4$ \\
\hline
\end{tabular}

zone 2 in 10, at zone 1 in 8 , and in both zones in 9 (Table 3). Nodal upstaging rate, in relation to reason for classification as potentially advanced NSCLC, were (I) primary tumor SUV >3 (52.3\%); (II) lymph node laterality by PET-CT (58.3\%); (III) preoperative CEA $>5 \mathrm{ng} / \mathrm{dL}$ (77.2\%); and (IV) suspected nodal metastasis during surgery (89.2\%). Furthermore, nodal upstaging was more frequent for tumors located in the left lower lobe $(\mathrm{P}=0.03)$ (Table 4).

\section{Outcomes}

Median duration of follow up was 52 months, and 32 (42.7\%) patients received adjuvant chemotherapy. Oral 5 -fluorouracil was administered to 9 pathological stage I
Table 2 Surgical variables

\begin{tabular}{|c|c|c|}
\hline Variable & Total $n$ & $\begin{array}{c}\text { Value/ } \\
\text { percentage }\end{array}$ \\
\hline \multicolumn{3}{|l|}{ Surgical procedure } \\
\hline LUL & 57 & $76 \%$ \\
\hline LLL & 15 & $20 \%$ \\
\hline Segmentectomy & 3 & $4 \%$ \\
\hline Operative time (min), median [range] & 75 & 227 [137-407] \\
\hline Blood loss (mL), median [range] & 75 & $120[0-700]$ \\
\hline Total LN removed, mean \pm SD & 75 & $32.7 \pm 12.9$ \\
\hline $\begin{array}{l}\text { Duration of hospital stay (days), } \\
\text { median [range] }\end{array}$ & 75 & $12[7-132]$ \\
\hline \multicolumn{3}{|l|}{ Complication } \\
\hline Hoarseness & 11 & $15 \%$ \\
\hline Arrhythmia & 7 & $9 \%$ \\
\hline Pneumonia & 2 & $3 \%$ \\
\hline SMA emboli & 1 & $1 \%$ \\
\hline Empyema & 1 & $1 \%$ \\
\hline Chylothorax & 1 & $1 \%$ \\
\hline Air leak $>7$ days & 1 & $1 \%$ \\
\hline
\end{tabular}

patients and to $1 \mathrm{p}$-stage II patient. The other 22 patients (1, 4, and 17 patients with p-stage I/II/III cancer) received platinum-doublet chemotherapy. Recurrence or metastasis during follow-up was noted in 29 patients ( $8 \mathrm{pN} 0$ patients, $2 \mathrm{pN} 1$ patients, $18 \mathrm{pN} 2$ patients, and $1 \mathrm{pN} 3$ patient). Only 2 patients developed locoregional lymph node recurrence around the carina.

OS rates at 3 and 5 years were $91.3 \% / 81.5 \%$ (Figure $2 A$ ). Survival at 3 and 5 years, stratified by p-stage, was $92.2 \% / 88.4 \%$ for p-stage I, $100.0 \% / 60.0 \%$ for p-stage II, and $87.7 \% / 81.0 \%$ for p-stage III, respectively (Figure 2B). Survival at 3 and 5 years, stratified by regional nodal staging, was $92.2 \% / 88.4 \%$ for $\mathrm{pN} 0,100.0 \% / 60.0 \%$ for $\mathrm{pN} 1$, and $87.4 \% / 80.7 \%$ for pN2 (Figure $2 C$ ).

\section{Discussion}

VATS is a common surgical strategy for small nodenegative lung cancers. Some previous studies reported that outcomes for these cancers under VATS were not inferior to 
Table 3 Number of dissected lymph nodes and metastatic lymph nodes in pN2 27 patients

\begin{tabular}{|c|c|c|c|c|c|}
\hline $\begin{array}{l}\text { Region of metastatic } \\
\text { lymph nodes }\end{array}$ & Patients & \multicolumn{2}{|c|}{ Number of dissected lymph nodes } & \multicolumn{2}{|c|}{ Number of metastatic lymph nodes } \\
\hline Zone 1 only & 8 & 7.5 [3-20] & 16 [2-25] & $1.5[1-8]$ & - \\
\hline Zone 2 only & 10 & $4.5[0-15]$ & 22 [9-32] & - & $3[1-9]$ \\
\hline Both zones 1 and 2 & 9 & $10[3-17]$ & $12[5-27]$ & $1[1-10]$ & $2[1-9]$ \\
\hline
\end{tabular}

Data are presented as median [range]. Zone 1, stations 3a, 5, and 6; zone 2, stations $2 \mathrm{~L}, 4 \mathrm{~L}$ and 7.

Table 4 Variables associated with nodal upstaging of cN0 NSCLC

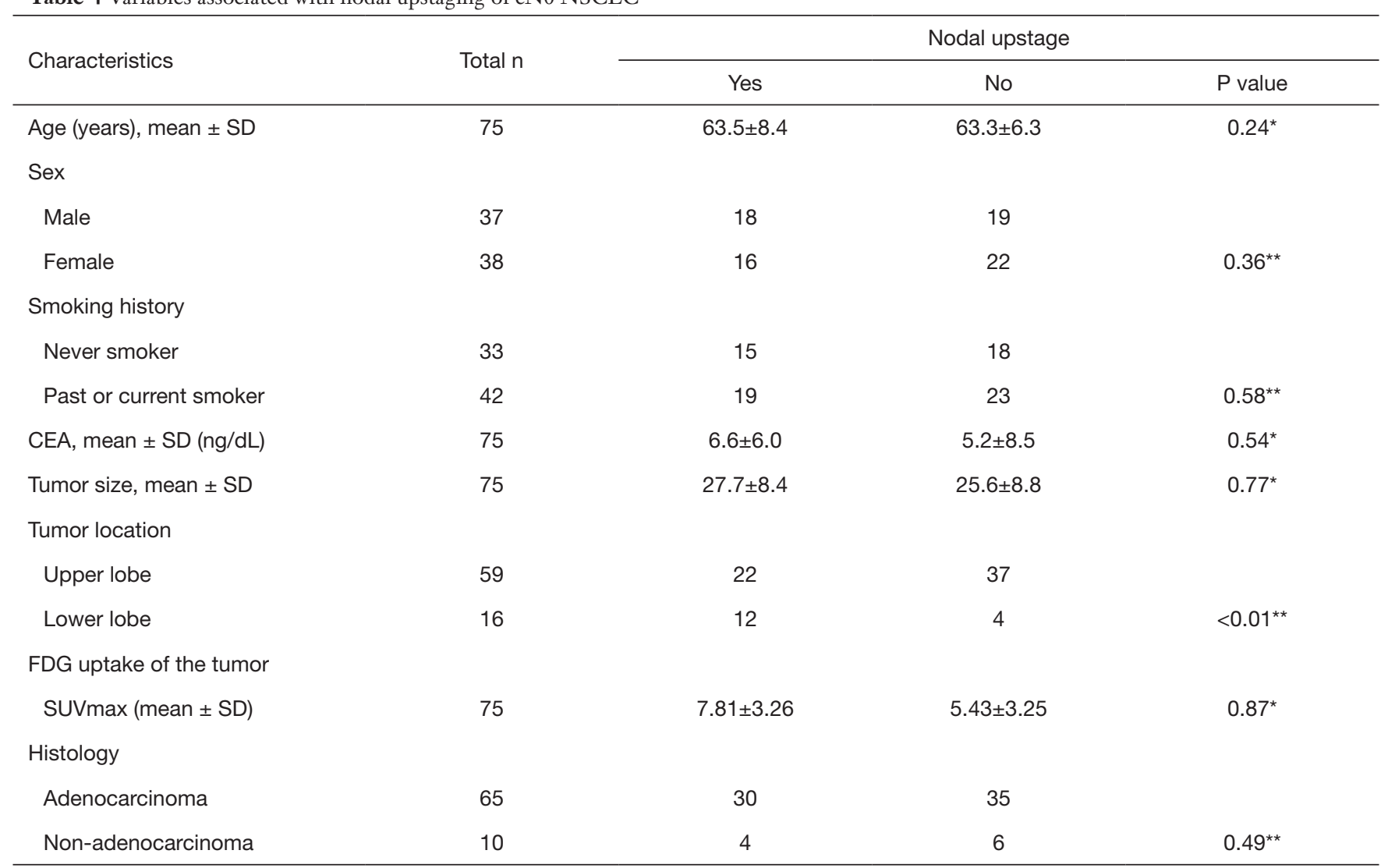

${ }^{*}$, student $t$-test; ${ }^{* \star}$, chi-square test. NSCLC, non-small cell lung cancer.

those for open thoracotomy, even when node involvement was unexpected. These include small clinical N0, pN2 lung cancers. Such cancers are associated with better outcomes than clinical and pathological N2 cancers, for which surgery is contraindicated (5). Some of the present patients might have been classified as clinical N2 cases if they had undergone invasive node biopsy via bronchoscopy or mediastinoscopy. Diagnostic accuracy of invasive biopsy for PET-positive lymph node is low even when the lymph nodes are over $1 \mathrm{~cm}(6)$, so necessity of preoperative invasive evaluation of PET-positive small $(<1 \mathrm{~cm})$ lymph node is controversial. Therefore, invasive biopsy was not performed for the present patients, even when PET-CT suggested node involvement.

The greatest obstacles in predicting surgical outcome after LND are that node involvement cannot always be definitively diagnosed without systemic LND and that stage migration substantially affects prognosis. Some previous 

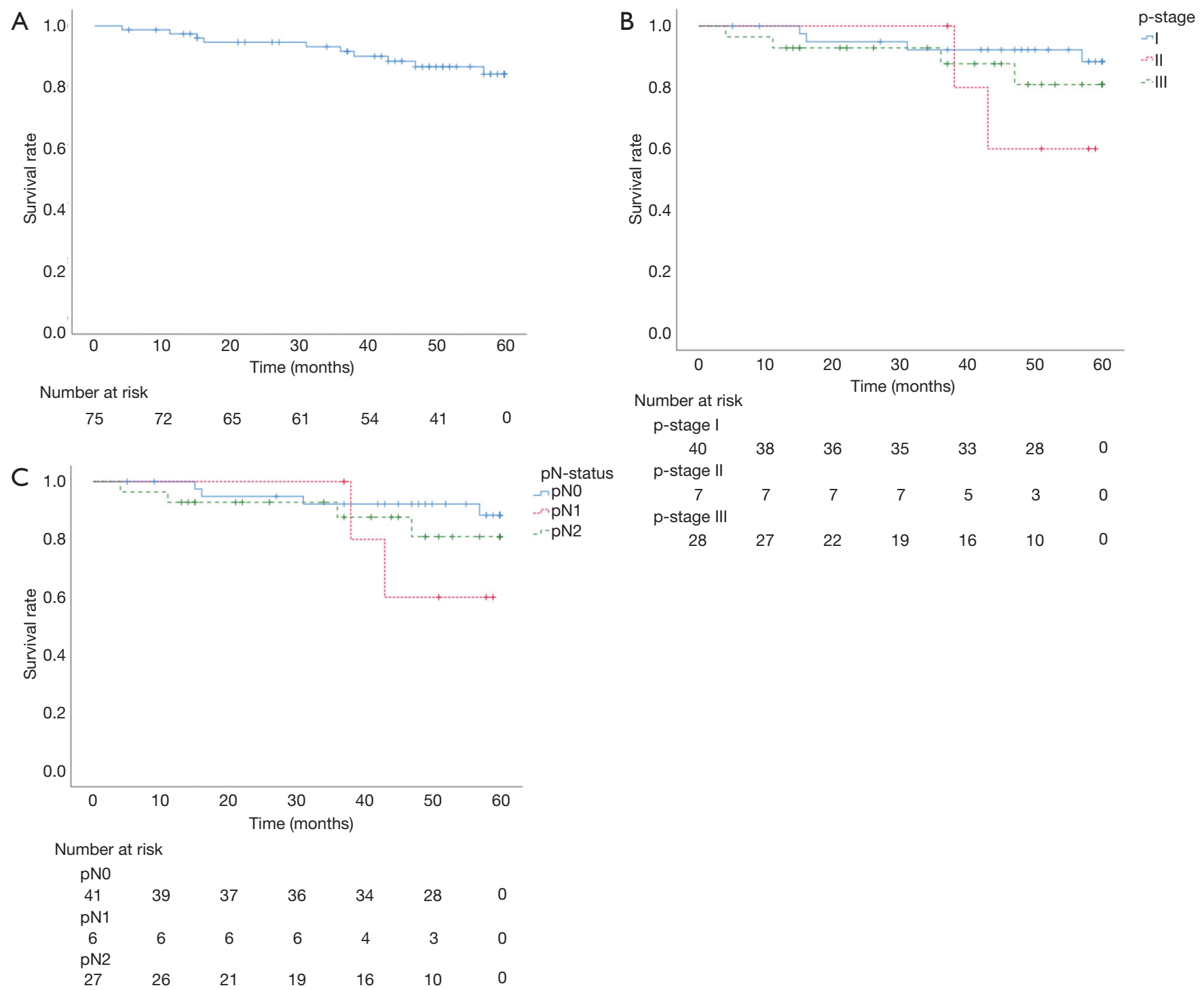

\begin{tabular}{|c|c|c|c|c|c|c|}
\hline 40 & 38 & 36 & 35 & 33 & 28 & 0 \\
\hline \multicolumn{7}{|c|}{ p-stage II } \\
\hline 7 & 7 & 7 & 7 & 5 & 3 & 0 \\
\hline \multicolumn{7}{|c|}{ p-stage III } \\
\hline 28 & 27 & 22 & 19 & 16 & 10 & 0 \\
\hline
\end{tabular}

Figure 2 Survival curves of the patients undergoing left mediastinal node dissection after arterial ligament transaction via video-assisted thoracoscopic surgery. (A) Overall survival; (B) overall survival, stratified by pathological stage (p-stage); (C) overall survival, stratified by pathologically confirmed regional node stage.

studies found no difference in survival between lymph node sampling and systematic LND (7-9) in node-negative patients. In this study we noted that, in addition to being beneficial for precise diagnosis of nodal micrometastases in potentially node-positive NSCLC, systematic LND also yielded outcomes that were much better than those for node-positive patients in previous reports. Extended LND may improve outcomes for patients with early lung cancer and radiologically undetectable nodal micrometastases.

Surgical procedures, and thus stage migration, vary by center. Using VATS, Merritt et al. reported dissection of $9.9 \pm 0.8$ nodes for early-stage NSCLC (10). Similarly, Denlinger $e t$ al. reported dissection of $8.9 \pm 5.2$ nodes with VATS (11). As compared with techniques used in previous studies, the present ALT technique enables en bloc dissection of three times as many lymph nodes, which increased the nodal upstaging rate (Table 5) (10,12-14). Dissection of fewer than 11 lymph nodes is associated with a poor prognosis (15); thus, extended mediastinal LND might improve outcomes for left NSCLC with micrometastases. However, adapting LND via ALT is not practical for all patients with left NSCLC. Hattori et al. reported that 
Table 5 Nodal upstage and number of dissected lymph nodes

\begin{tabular}{lcccc}
\hline First author & Number of patients & Procedure & Number of nodes dissected & Upstaging rate (\%) \\
\hline Our study & 84 & VATS & $37.2 \pm 12.9$ & 45.3 \\
Merritt (10) & 60 & VATS & $9.9 \pm 0.8$ & 10 \\
& 69 & Open & $14.7 \pm 1.3$ & 24.6 \\
Licht (12) & 717 & VATS & $4.57 \pm 1.42$ & 5.9 \\
& 796 & Open & $4.51 \pm 0.37$ & 13.2 \\
Reichert (13) & 77 & VATS & $19.57 \pm 0.99$ & 16.9 \\
Moon (14) & 634 & Unclassified & $16.6 \pm 7.8$ & 9.3 \\
\hline
\end{tabular}

VATS, video-assisted thoracoscopic surgery.

presence of solid carcinoma, a CEA level $>5 \mathrm{ng} / \mathrm{dL}$, and a maximum SUV $>5$ predicted unexpected nodal involvement in clinical stage I NSCLC (16). Extended mediastinal LND under ALT might be suitable for these potentially nodepositive patients.

Lymphadenectomy for the left thorax is often technically difficult because the thoracic aorta, left main pulmonary artery, and arterial ligament conceal the paratracheal and subcarinal spaces. This deep narrow space is difficult to see and limits sharing of information between surgeons under direct view via open thoracotomy. However, when using our ALT technique via VATS, this deep concealed space is readily visualized and shared by video monitor, which enables easier en bloc dissection of the carinal zone (paratracheal and subcarinal lymph nodes), as shown in Figure 1. Nodal upstaging after extended LND was more frequent in patients with cancer of the left lower lobe, because node involvement around the carina through station 7 was susceptible to metastasis (17).

The incidence of recurrent nerve paralysis in the present patients was comparatively high (14.6\%). Most patients with recurrent nerve paralysis were treated in the first half of the study period, and thermal damage to the recurrent nerve by an ultrasonic energy device was the main reason for paralysis. However, $72.7 \%$ of the patients with hoarseness recovered. The energy device was not used during dissection around the recurrent nerve in the second half of the study period, as confirmed in Figure 1.

This study had some limitations, the most important of which is that it was a one-arm retrospective study at a single institution and was therefore unable to assess the true effectiveness of extended LND via ALT. A second limitation is that the stage migration effect could have affected rates of nodal upstaging and survival, because ALT was performed for selected patients with suspected advanced cancer. Despite these limitations, the excellent survival rate among node-positive patients suggests that extended LND with ALT is beneficial when radiologically negative but potentially node-positive cancer is diagnosed during surgery. Prospective clinical studies are necessary in order to clarify the survival effect of extended LND via ALT by analysis of LND, with or without ALT.

\section{Acknowledgements}

None.

\section{Footnote}

Conflicts of Interest: The authors have no conflicts of interest to declare.

Ethical Statement: This study was approved by the institutional review board of Jichi Medical University (No. 17-056). Informed consent was obtained in the form of optout on a website for the study.

\section{References}

1. Ettinger DS, Bepler G, Bueno R, et al. Non-small cell lung cancer clinical practice guidelines in oncology. J Natl Compr Canc Netw 2006;4:548-82.

2. Lardinois D, De Leyn P, Van Schil P, et al. ESTS guidelines for intraoperative lymph node staging in non-small cell lung cancer. Eur J Cardiothorac Surg 2006;30:787-92.

3. Goldstraw P, Crowley J, Chansky K, et al. The IASLC Lung Cancer Staging Project: proposals for the revision 
of the TNM stage groupings in the forthcoming (seventh) edition of the TNM Classification of malignant tumours. J Thorac Oncol 2007;2:706-14.

4. Shibano T, Tsubochi H, Tetsuka K, et al. Left mediastinal node dissection after arterial ligament transaction via videoassisted thoracoscopic surgery (the top of images shows the cranial side). Asvide 2018;5:931. Available online: http:// www.asvide.com/article/view/29193

5. Zhong C, Yao F, Zhao H. Clinical outcomes of thoracoscopic lobectomy for patients with clinical N0 and pathologic N2 non-small cell lung cancer. Ann Thorac Surg 2013;95:987-92.

6. Lilo MT, Allison DB, Younes BK, et al. The critical role of EBUS-TBNA cytology in the staging of mediastinal lymph nodes in lung cancer patients: A correlation study with positron emission tomography findings. Cancer Cytopathol 2017;125:717-725.

7. Darling GE, Allen MS, Decker PA, et al. Randomized trial of mediastinal lymph node sampling versus complete lymphadenectomy during pulmonary resection in the patient with N0 or N1 (less than hilar) non-small cell carcinoma: results of the American College of Surgery Oncology Group Z0030 Trial. J Thorac Cardiovasc Surg 2011;141:662-70.

8. Wu N, Yan S, Lv C, et al. Does an extended mediastinal lymphadenectomy improve outcome after $\mathrm{R} 0$ resection in lung cancer? Chin J Cancer Res 2014;26:183-91.

9. Sugi K, Nawata K, Fujita N, et al. Systematic lymph node dissection for clinically diagnosed peripheral non-smallcell lung cancer less than $2 \mathrm{~cm}$ in diameter. World J Surg 1998;22:290-4; discussion 294-5.

Cite this article as: Shibano T, Tsubochi H, Tetsuka K, Yamamoto S, Kanai Y, Minegishi K, Endo S. Left mediastinal node dissection after arterial ligament transection via videoassisted thoracoscopic surgery for potentially advanced stage I non-small cell lung cancer. J Thorac Dis 2018;10(12):64586465. doi: 10.21037/jtd.2018.11.86
10. Merritt RE, Hoang CD, Shrager JB. Lymph node evaluation achieved by open lobectomy compared with thoracoscopic lobectomy for N0 lung cancer. Ann Thorac Surg 2013;96:1171-7.

11. Denlinger CE, Fernandez F, Meyers BF, et al. Lymph node evaluation in video-assisted thoracoscopic lobectomy versus lobectomy by thoracotomy. Ann Thorac Surg 2010;89:1730-5; discussion 1736.

12. Licht PB, Jørgensen OD, Ladegaard L, et al. A national study of nodal upstaging after thoracoscopic versus open lobectomy for clinical stage I lung cancer. Ann Thorac Surg 2013;96:943-9; discussion 949-50.

13. Reichert M, Steiner D, Kerber S, et al. A standardized technique of systematic mediastinal lymph node dissection by video-assisted thoracoscopic surgery (VATS) leads to a high rate of nodal upstaging in early-stage non-small cell lung cancer. Surg Endosc 2016;30:1119-25.

14. Moon Y, Lee KY, Kim KS, et al. Clinicopathologic correlates of postoperative N1 or N2 nodal upstaging in non-small cell lung cancer. J Thorac Dis 2016;8:79-85.

15. Tantraworasin A, Saeteng S, Siwachat S, et al. Impact of lymph node management on resectable non-small cell lung cancer patients. J Thorac Dis 2017;9:666-74.

16. Hattori A, Suzuki K, Matsunaga T, et al. Is limited resection appropriate for radiologically "solid" tumors in small lung cancers? Ann Thorac Surg 2012;94:212-5.

17. Shimada Y, Saji H, Kakihana M, et al. Retrospective analysis of nodal spread patterns according to tumor location in pathological N2 non-small cell lung cancer. World J Surg 2012;36:2865-71. 